\section{Repercusiones de "ObamaCare" para la reforma sanitaria en Chile}

\author{
RAÚL ALLARD SOTO
}

\section{"ObamaCare": effects for the health reform in Chile}

Health care raises structural issues in a democratic society, such as the role assigned to the central government in the management of health risk and the redistributive consequences generated by the implementation of social insurance. These are often cause of strong political controversy. This paper examines the United States of America health reform, popularly known as "ObamaCare". Its three main elements, namely individual mandate, creation of new health insurance exchanges, and the expansion of Medicaid, generated a redistribution of health risks in the insurance market of that country after almost a century of frustrated legislative efforts to guarantee minimum universal coverage. The article proposes that a change of this magnitude in the United States will produce effects in a forthcoming parliamentary discussion on the health reform in Chile, which still maintains a highly deregulated private health system.

(Rev Med Chile 2019; 147: 103-106)

Key words: Chile; Insurance, Health; Patient Protection and Affordable Care Act; Universal Health Insurance; United States.
'Departamento de Ciencias Jurídicas de la Universidad de La Frontera. Temuco, Chile.

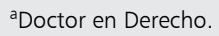

El autor declara no tener conflictos de intereses que declarar.

Financiado por la Universidad de La Frontera, Proyecto DI18-0077.

Correspondencia a:

Profesor Raúl Allard Soto

Facultad de Ciencias Jurídicas y

Empresariales, Universidad

de La Frontera.

Francisco Salazar №01145.

Temuco, Chile.

raul.allard@ufrontera.cl
$\mathrm{E}$ 1 derecho a la salud constituye una preocupación ciudadana creciente y sostenida. En el centro de la discusión se encuentran las obligaciones que tiene el Estado en su protección y garantía frente a las progresivas expectativas de las personas por acceder a mayores y mejores prestaciones.

En nuestro país se ha ido imponiendo la idea de que el Estado debiera garantizar el acceso universal a cuidado sanitario apropiado en base a la necesidad de atención sanitaria. En 2010, en los Estados Unidos de Norteamérica (en adelante EE.UU.), con la reforma sanitaria del presidente Barack Obama, se legisló en esta dirección, cuya aplicación en Chile implicaría la transformación del sistema actual de seguros de salud en un sistema de seguridad social regido por los principios de universalidad, libre movilidad, no selección por ingreso, exclusiones por preexistencias o carencias y cobertura vitalicia ${ }^{1}$.

Esta reforma denominada "The Patient Protection and Affordable Care Act" (en adelante "Obama-
Care") merece ser analizada detenidamente, luego de casi un siglo de esfuerzos legislativos frustrados por garantizar un mínimo sanitario universal en EE.UU. ${ }^{2,3}$ y extraer de ella conclusiones relevantes para una eventual reforma en nuestro país.

Atendido que el cuidado de la salud plantea cuestiones estructurales en una sociedad democrática sobre el tipo de nación y de gobierno que sus ciudadanos resuelven alcanzar, "ObamaCare" abordó el rol asignado al gobierno central en la prevención, sustitución y dispersión de la administración del riesgo, objeto de fuerte controversia ${ }^{5}$ política, por cuanto qué riesgo abordar y cómo hacerlo, necesariamente genera consecuencias redistributivas ${ }^{6}$. La acalorada discusión sobre la reforma de salud de la última década entre los partidos republicano y demócrata así lo atestiguan.

En su forma reestructurada, nadie puede ser rechazado por un seguro de salud ${ }^{7}$, dejando de ser un producto diseñado para preservar la rentabilidad a través de un proceso de selección adversa $\sin$ frenos, a una industria regulada, cuya fuerza y 
estabilidad a largo plazo se consideran esenciales para el interés público ${ }^{8}$. En contrapartida, impone impuestos, responsabilidades o sanciones a terceros en condiciones de mitigar o absorber mejor el riesgo ${ }^{6}$.

Un cambio de esta envergadura en EE.UU., muy probablemente debería producir efectos en nuestro país, que aún mantiene un sistema privado de salud fuertemente desregulado ${ }^{1,9}$. Como un aporte en esa dirección, abordaremos primero la reforma de Massachusetts -antecedente directo de "ObamaCare"- para luego analizar los elementos distintivos de la reforma federal de salud; posteriormente se destacará la argumentación de la Corte Suprema de ese país, que determinó que la reforma federal se ajustaba a la Constitución, finalizando con consideraciones relevantes del proceso en EE.UU. de cara a una próxima discusión parlamentaria en nuestro país.

\section{El modelo de Massachusetts que inspira la reforma federal}

Una ventaja del sistema federal es que permite a los estados miembros de la federación servir como "laboratorios" para la experimentación de políticas públicas ${ }^{10}$. Bien lo sabía el fallecido senador demócrata Edward Kennedy, mientras instaba al gobierno federal a ampliar la cobertura del seguro de salud a todos los estadounidenses ${ }^{11}$, apoyó resueltamente al gobernador republicano de Massachusetts, Mitt Romney, impulsor de una reforma sanitaria en Massachusetts, aprobada en 2006 con amplia mayoría parlamentaria en ese estado $^{12}$. Al promulgar la ley, Romney dijo que esta proporcionaría a cada ciudadano de Massachusetts un seguro médico asequible y completo ${ }^{13}$.

La reforma incluyó tres componentes esenciales $^{12}$ :

(i) el "mandato individual" que exige a aquellos ciudadanos sin seguro médico obtener cobertura de salud, o en su reemplazo, pagar una multa;

(ii) la "creación de intercambios de seguros de salud" donde personas sin seguro pueden comprar cobertura médica ${ }^{14}$, fijando un intercambio de seguro de salud en línea denominado "Health Connector" 13,15 junto a un sistema de deducciones fiscales para ayudar a las personas a comprar un seguro ${ }^{13}$; iii) una "expansión de MEDICAID" -el programa federal que proporciona subsidios federales a los estados miembros de la federación para ofrecer seguros a ciudadanos de bajos ingresos- en Massachusetts, priorizando el ingreso a MEDICAID de niños hasta $300 \%$ de la línea de pobreza, mujeres embarazadas hasta $200 \%$ y los padres hasta $133 \%{ }^{12}$.

El modelo ayudó a reducir la proporción de personas sin seguro al más bajo de todos los estados de la nación. En 2010, más de 98\% de los residentes del Estado estaban asegurados, incluido $99,8 \%$ de todos los niños ${ }^{16}$.

\section{2. "ObamaCare": La reforma federal de salud}

"ObamaCare" constituye un programa de seguro a nivel nacional de carácter integral ${ }^{17,18}$, que configura un modelo híbrido de cobertura universal que utilizan muchos países europeos, al combinar la cobertura del empleador con cooperativas de compra, programas de atención a la pobreza, seguro gubernamental y regulación de los seguros de salud, de manera que todos los ciudadanos se encuentren cubiertos con algún tipo de seguro en una forma fiscalmente realista ${ }^{14}$. Para ello, adapta la reforma de Massachusetts al ámbito federal, en los siguientes términos:

i) el "mandato individual" como prerrequisito para la cobertura universal. Todas las personas sin seguro de salud deben obtener uno a contar del año 2014, o bien, proceder al pago de una multa $^{14}$, prohibiéndose las discriminaciones basadas en género y en condiciones preexistentes de salud. El rol que adopta no difiere esencialmente de la participación universal contemplada en otros acuerdos colectivos de distribución de riesgos en EE.UU., como la Seguridad Social o como MEDICARE ${ }^{19}$, el programa federal de seguro de salud que se extiende a prácticamente todos los ciudadanos de sesenta y cinco años o más;

ii) la "creación de nuevos intercambios de seguros de salud" a contar del año 2014, que contratan con aseguradores privados el establecimiento de tarifas grupales y agregan compradores de seguros individuales a los grupos de compras. De este modo, se reducen los costos administrativos para las compañías de seguros, se redu- 
cen las tasas para los individuos que compran seguros y permite que una persona obtenga el mismo apalancamiento de mercado que podría obtener un gran empleador que compre una póliza de grupo ${ }^{14}$; al mismo tiempo subsidia el costo de la cobertura para quienes ganan entre $133 \%$ y $400 \%$ de la línea de pobreza ${ }^{20}$;

iii) la "expansión de MEDICAID" al incluir ahora a todas las personas cuyos ingresos están por debajo de $138 \%$ del nivel federal de pobreza ${ }^{21}$, brindando atención médica subsidiada a millones de estadounidenses más pobres.

De esta manera, "ObamaCare" realiza el cambio más significativo al contrato social en EE.UU. desde la creación de "MEDICAID" y "MEDICARE" en la década de 1960-69, cumpliendo el sueño de los progresistas de la atención universal $\mathrm{y}$ asequible para todos ${ }^{22}$.

\section{La Corte Suprema de EE.UU. legitima constitucionalidad de "ObamaCare"}

De acuerdo con Balkin, cuando los cambios son grandes y el gobierno federal expande sus capacidades, son los tribunales federales los encargados de legitimarlos y garantizar que estos sean consistentes con la Constitución de EE.UU.22.

Desde su promulgación como ley federal, "ObamaCare" fue objeto de múltiples reclamaciones judiciales. Dos controversias emblemáticas dirigidas a derogarla en su integridad ${ }^{23}$ fueron resueltas finalmente por la Corte Suprema, declarando que "ObamaCare" se ajustaba a la Constitución de EE.UU. Ambas reclamaciones se encontraban motivadas, en lo fundamental, en el fastidio que generaba -en algunas personas, grupos de presión o gremios- la redistribución del ingreso en el nuevo régimen: sea porque el gobierno no debiera poder obligar a algunas personas a comprar un seguro que subsidie eficazmente la atención médica de los demás, o bien, porque la expansión de MEDICAID desviaba injustamente los dólares de los impuestos federales a las personas pobres y desplazaba el dinero hacia aquellos estados federados que concentran más gente pobre ${ }^{22}$.

En la histórica sentencia "NFIB v. Sebelius", de 2012, la Corte Suprema decidió que el mandato individual era constitucional al corresponder efectivamente a un impuesto dispuesto en otra forma, que el Congreso tenía el derecho de establecer ${ }^{14}$. Al justificar su trascendental fallo judicial, el Presidente John Roberts argumentó que la Corte tenía una "reticencia general para invalidar los actos de los líderes electos de la Nación"24. Posteriormente, en 2015, la Corte Suprema en "King v. Burwell" determinó que "ObamaCare" autorizaba la entrega de "créditos fiscales federales para estadounidenses elegibles que viven no solo en estados con sus propios intercambios, sino también en los 34 estados con mercados federales" 25 .

\section{Consideraciones para la reforma sanitaria en Chile}

La ley federal de reforma de la salud de EE.UU. fue redistributiva y sus 3 elementos primordiales mandato individual; creación de nuevos intercambios de seguros de salud y la expansión de MEDICAID- se unieron para generar una redistribución de los riesgos sanitarios en el mercado de seguros de ese país, constituyendo, en nuestro concepto, su repercusión de mayor trascendencia de cara a la reforma sanitaria pendiente en nuestro país.

Una redistribución de los riesgos debe formar parte importante de una próxima discusión parlamentaria en Chile, atendido el desequilibrio reinante en nuestra legislación entre el subsistema público -FONASA- y el subsistema privado -ISAPRES- de salud, diseñado para que FONASA asegure a la población con más riesgo sanitario y menos ingresos en Chile (13,5 millones de afiliados), mientras las ISAPRES afilian a la población con más recursos, más joven y con menos riesgo sanitario $(3,5 \text { millones de personas })^{9}$. Esto afecta el desarrollo de un sistema de salud equilibrado.

Alcanzar una ley que distribuya equitativamente los riesgos sanitarios puede implicar la provisión de una cobertura mínima de salud universal para todos los ciudadanos - como la diseñada por "ObamaCare"- que prohíba las discriminaciones basadas en género, edad y en condiciones preexistentes de salud que subsisten en el sistema privado de salud de nuestro país desde su instalación en la década de 1980-89.

Por último, la reforma de salud de EE.UU. ejemplifica que la redistribución de los riesgos sanitarios no impide una provisión mixta de prestadores públicos y privados que asegure la 
apropiada prestación del servicio compatible con el propósito de un seguro social: la distribución de los recursos a lo largo de la vida de los individuos y la redistribución desde las familias que no han incurrido en los riesgos asegurados hacia aquellas que sí han incurrido en ellos, asegurando la protección del interés general y colectivo de la población. Chile tiene la oportunidad de seguir la misma dirección.

\section{Referencias}

1. Zúñiga Fajuri A. Propuesta para la reforma definitiva del sistema privado de salud chileno. Rev Med Chile 2014; 142: 501-5.

2. Graetz M, Mashaw J. Constitutional Uncertainty and the Design of Social Insurance. En: Persily N, Metzger G, Morrison T, Editores, The Health Care Case: The Supreme Court's Decision and its Implications. New York, United States of America: Oxford University Press; 2013. p. 300-314.

3. Duggin S. From the New Deal to the New Healthcare: A New Deal Perspective on King v. Burwell and the Crusade Against the Affordable Care Act. University of Miami Business Law Review 2015; 23: 317-39.

4. Coyle M. The Roberts court: the struggle for the constitution. Simon \& Schuster Paperbacks, New York, 2014, p. 335.

5. Baker T, Moss D. Government as Risk Manager. En: Moss D, Cisternino J, Editores, New Perspectives on Regulation. Massachusetts, United States of America: The Tobin Project, 2009. p. 87-109.

6. Huq A. Federalism, Liberty, and Risk in NFIB v. Sebelius. En: Malani A, Schill M, Editores, The Future of Healthcare Reform in the United States. Chicago, United States of America: The University of Chicago Press; 2015. p. 32-49.

7. Hall M. Health Care Reform - What went wrong on the Way to the Courthouse. N Engl J Med 2011; 364 (4): 295-7.

8. Priest AJG. Possible adaptation of public utility concepts in the health care field. Law Contemp Probl 1970; 35: 839-48.

9. Allard R, Hennig M, Galdamez L. El derecho a la salud y su (des)protección en el Estado subsidiario. Estudios Constitucionales 2016; 14 (1): 95-138.

10. Walton H, Smith R, Wallace S. American Politics and the African American Quest for Universal Freedom. Routledge, Eighth Edition, New York, 2017, p. 32.

11. Kennedy E. The Role Of The Federal Government In
Eliminating Health Disparities. Health Affairs 2005; 24 (2): 452-8.

12. Thompson F. Medicaid Politics: Federalism, Policy Durability and Health Reform. Georgetown University Press, Washington DC, 2012, p.167-73.

13. Dawes D. 150 Years of Obamacare. John Hopkins University Press, Baltimore, 2016, p. 180.

14. Engel J. Unaffordable: American healthcare from Johnson to Trump. The University of Wisconsin Press, Wisconsin; 2018, p. 197-207.

15. Rebitzer J. The Affordable Care Act and Commercial Health Insurance Markets. En: Malani A, Schill M, Editores, The Future of Healthcare Reform in the United States. Chicago, United States of America: The University of Chicago Press; 2015. p. 313-28.

16. Quadagno J. The Transformation of Medicaid from Poor Law Legacy to Middle-Class Entitlement?. En: Cohen A, Colby D, Wailoo K, Zelizer J, Editores, Medicare and Medicaid at 50: America's entitlement programs in the age of affordable care. Oxford University Press, New York; 2015. p. 77-94.

17. Ginsburg R, Hartnett M, Williams W. My own words. New York, Simon \& Schuster; 2016. p. 309.

18. Oberlander J. Beyond Repeal-The future of Health Care Reform. N Engl J Med 2010; 363: 2277-9.

19. Avsar R. A Rawlsian Defense of the Individual Mandate: The "Collective Asset" Approach. Review of Social Economy 2015; 73 (2): 146-53.

20. Harrington S. U.S. Health-Care Reform: The Patient Protection And Affordable Care Act. J Risk Insur 2010; 77 (3): 703-8.

21. Cohen A, Colby D, Wailoo K, Zelizer J. Conclusion: the world that Medicare and Medicaid made. En: Cohen A, Colby D, Wailoo K, Zelizer J, Editores, Medicare and Medicaid at 50: America's entitlement programs in the age of affordable care. Oxford University Press, New York; 2015. p. 333-40.

22. Balkin J. The Court Affirms the Social Contract. En: Persily N, Metzger G, Morrison T, Editores, The Health Care Case: The Supreme Court's Decision and It's Implications. Oxford University Press, New York; 2013. p. 11-6.

23. Tschann M, Soon R. Contraceptive Coverage and the Affordable Care Act. Obstet Gynecol Clin North Am. 2015; 42(4): 605-17.

24. National Federation of Independent Business, et al. v. Sibelius, Secretary of Health and Human Services, et al. June 28, 2012, p. 6.

25. Ariane de Vogue y Jeremy Diamond, "Supreme Court Saves Obamacare", CNN, 25 de junio, 2015. En: http:// www.cnn.com/2015/06/25/politics/supreme-court-ruling-obamacare/ [accedido el 8 de agosto de 2018]. 